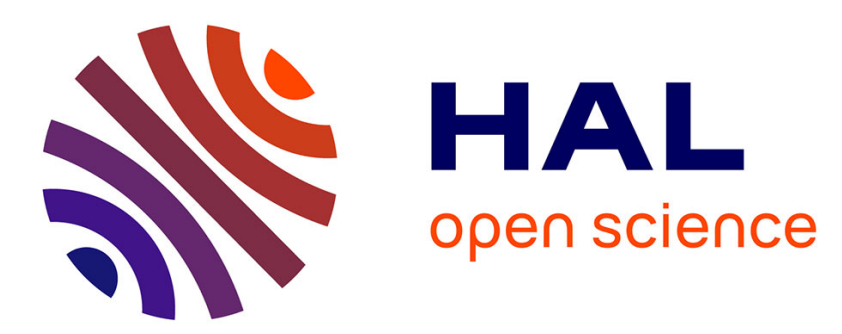

\title{
Using more channels can be detrimental to the global performance in interference networks
}

Chao Zhang, Samson Lasaulce, Elena Veronica Belmega

\section{To cite this version:}

Chao Zhang, Samson Lasaulce, Elena Veronica Belmega. Using more channels can be detrimental to the global performance in interference networks. IEEE International Conference on Communication Workshop (ICCW), Jun 2015, London, United Kingdom. 10.1109/ICCW.2015.7247169 . hal01272508

\section{HAL Id: hal-01272508 \\ https://hal.science/hal-01272508}

Submitted on 15 Feb 2016

HAL is a multi-disciplinary open access archive for the deposit and dissemination of scientific research documents, whether they are published or not. The documents may come from teaching and research institutions in France or abroad, or from public or private research centers.
L'archive ouverte pluridisciplinaire HAL, est destinée au dépôt et à la diffusion de documents scientifiques de niveau recherche, publiés ou non, émanant des établissements d'enseignement et de recherche français ou étrangers, des laboratoires publics ou privés. 


\section{Using more channels can be detrimental to the global performance in interference networks}

\author{
Chao Zhang \\ L2S \\ CNRS-Centrale Supelec-Univ. Paris Sud \\ 91190 Gif-sur-Yvette, France
}

\author{
Samson Lasaulce \\ L2S \\ CNRS-Centrale Supelec-Univ. Paris Sud \\ 91190 Gif-sur-Yvette, France
}

\author{
E. Veronica Belmega \\ ETIS / ENSEA - UCP - CNRS \\ 95014 Cergy-Pontoise, France
}

\begin{abstract}
We study distributed interference networks in which the transmitting-receiving pairs (or users) can communicate over several orthogonal channels. The network is said to be decisionwise distributed because each transmitter is assumed to choose the way it spreads its power over the available channels to maximize its individual rate. The main contribution of this paper is to prove that, depending on the network parameters, allowing every transmitter to use several channels simultaneously instead of a single one may lead to a global performance degradation in terms of the sum-rate at Nash equilibrium. In distributed networks, the Nash equilibria have the potential to be implemented with local knowledge and by low-complexity iterative or learning processes. Performance degradation is typically observed when the signal-to-noise ratio (SNR) is sufficiently high. The existence of such scenarios is proved analytically for networks composed of arbitrary number of users and channels and for large networks (of many users). Simulations confirm our analytical results in the low and high SNR regimes but also illustrate this phenomenon for intermediate SNR values.
\end{abstract}

\section{INTRODUCTION}

Wireless networks in which transmitters have to take decisions on how to use radio resources in an autonomous manner capture an increasing interest. We will refer to this type of wireless networks as distributed or decentralized wireless networks. An example of such a network is given by small cell networks (SCNs) [2]. Indeed, one way of boosting data rates in cellular networks is to deploy a large number of small base stations that cannot be controlled by a single central entity and therefore have to be nearly autonomous in terms of managing radio resources. Another important example of such networks is WiFi networks: each access point has to select the operating channel or band without the assistance of a central entity. In both SCNs and WiFi networks, it may seem quite obvious that the more channels the transmitters will use the higher the data rates will be. In this respect, the startup codeon [1] promotes a software solution which allows channel bundling to be implemented in WiFi networks, i.e., several WiFi channels are exploited simultaneously instead of one as done currently.

While the benefits in terms of data rate for a point-topoint communication between a (multi-channel) WiFi access point and a (multi-channel) WiFi user terminal are well understood, a question may arise about the global benefits when several access point-user terminal pairs operate in the multi-channel mode in the presence of interference. Similarly, $5 \mathrm{G}$ cellular phones are expected to exploit several technologies simultaneously or a single technology using multiple channels or bands at a time. If the system is centralized, allowing every transmitter to exploit more channels or bands "necessarily" leads to a potentially better global performance, say in terms of network sum-rate. However, if we consider a distributed network where transmitters perform individual or local maximization operations, it is not clear whether allowing them to use more channels will lead to a better global performance. Providing elements of response to this question is precisely the purpose of this paper.

To address the raised issue, we exploit one of the classical models of interference networks which is the parallel interference channel (PIC). This model represents a communication scenario in which several transmitter-receiver pairs communicate through a common set of orthogonal channels. While the problem of distributed power allocation in PICs has attracted a lot of attention from the communications community (the corresponding line of works was to a large extent pioneered by [11]), the problem of distributed channel selection in PICs has only been treated analytically in a relatively very small number of papers.

The closest works to the present paper are [6][9]. In [6], the authors focus on the Nash equilibrium existence and uniqueness problems of the following power allocation and channel selection ${ }^{1}$ games: the considered PIC is assumed to comprise two transmitter-receiver pairs and two channels; for each channel realization, each transmitter chooses its power allocation vector or selects its channel in order to "maximize" its individual data rate. The problem of comparing the global performance of the two scenarios in terms of power allocation policies is not tackled. In [9], the equilibrium analysis is treated in detail in the case of the parallel multiple access channel, which is a special case of the PIC. The problem of comparing the global performance in terms of sum-rate of distributed power allocation with the one of channel selection is introduced but not developed. Motivated by the practical importance of this comparison, the aim of the present work is to provide more general analytical and simulation results in PIC. Our interesting results provide a better understanding on how to use the spectrum in interference networks.

\footnotetext{
${ }^{1}$ Note that selecting a channel corresponds to allocating all the available power to a single channel.
} 
The paper is structured as follows. In Section II, the comparison of performance problem is formulated. In Section III, we provide several new analytical results which allow one to better characterize the global performance degradation phenomenon induced by using multiple channels in distributed interference networks. Section IV is dedicated to numerical results. In particular, simulations show that the global performance degradation phenomenon observed for small and large networks can also be observed for networks of intermediate sizes (e.g., for four transmitter-receiver pairs).

\section{MODEL AND PROBLEM FORMULATION}

Consider a wireless communications system, which comprises $K$ transmitter-receiver pairs. Each transmitter wants to communicate with its respective receiver. More precisely, transmitter $k \in \mathcal{K}, \mathcal{K}=\{1, \ldots, K\}$, has to allocate its available power among $N \geqslant 1$ orthogonal channels or frequency bands to maximize its own transmission rate (individual utility)

$$
u_{k}\left(p ; g_{k}\right)=\sum_{n=1}^{N} \log _{2}\left(1+\frac{g_{k k}^{n} p_{k}^{n}}{\sigma^{2}+\sum_{\ell \neq k} g_{\ell k}^{n} p_{\ell}^{n}}\right)
$$

where $p_{k}^{n}$ is the power transmitter $k$ allocates to band $n$, $p=\left(p_{1}^{1}, \ldots, p_{1}^{N}, \ldots, p_{K}^{1}, \ldots, p_{K}^{N}\right), g_{k \ell}^{n} \geqslant 0$ is the channel gain associated with the link from transmitter $k$ to receiver $\ell$ over band $n, g_{k}=\left(g_{1 k}^{1}, \ldots, g_{K k}^{1}, \ldots, g_{1 k}^{N}, \ldots, g_{K k}^{N}\right)$, and $\sigma^{2}$ accounts for the thermal noise. In our analysis, block fading channels are assumed. The channel gain $g_{k \ell}^{n}$ is assumed to be constant over a block and to be the realization of a random variable whose probability density function is given by: $f_{k \ell}^{n}(x)=\frac{1}{\gamma_{k \ell}^{n}} \exp \left(-\frac{x}{\gamma_{k \ell}^{n}}\right)$ for $x \geqslant 0$ and $f_{k \ell}^{n}(x)=0$ for $x<0$. Additionally, all channel gains are assumed to be drawn independently.

As one of our motivations is to assess the global network performance in terms of sum-utility, it might seem surprising that maximizing a individual utility function is considered as the objective assigned to a transmitter. This choice can be motivated by many arguments but we will just mention a few of them. Note that even if there were a central node that could control the whole vector $p$ and would have global channel state information (CSI) $g=\left(g_{1}, \ldots, g_{K}\right)$, it wouldn't be able to perform the direct maximization of the sum-utility w.r.t. $p$. Indeed, the corresponding problem is very difficult from an optimization point of view, but also from a computational point of view if exhaustive search over partitioned spaces is performed. Therefore, even if the network is devised by a single designer, there is a high interest in considering individual utilities with partial or local control of the variables. Of course, when such a central node is not available, considering individual utilities is much more compatible with the fact that, in general, transmitters can only perform partial control and have only partial or local CSI.

For a classical optimization problem which involves a single function and full control of its variables, the notion of optimality is clear. However, in the presence of multiple decision makers that have partial control of these variables, the very meaning of optimality is unclear. Indeed, the optimal decision for transmitter $k$ depends on the decisions made by the other transmitters. This is one of the reasons why other solution concepts than optimality need to be considered in such a setting. A fundamental solution concept is the notion of Nash point or equilibrium. As very well illustrated by the iterative water-filling algorithm (IWFA) [11], which converges $^{2}$ to a Nash equilibrium (NE), one of the major assets of the $\mathrm{NE}$ is that it may be implemented with local knowledge only and reached through low complexity iterative or learning procedures (see e.g., [5]). This is why we consider, in almost the entire paper, the performance of the network at equilibrium. The procedures or algorithms to reach $\mathrm{NE}$ are not addressed here. Before introducing an NE, we first define explicitly the strategic-form games.

The strategic-form game of interest consists of a triplet: the set of decision makers corresponding to $\mathcal{K}$ here; their strategy sets that contain all their possible choices and are the power allocation sets; and their utility functions that depend on their own choices but also on the others' choices and are defined here below. We consider three different scenarios with respect to the set of power allocation vectors allowed to each transmitter. The considered utility functions are given by (1) but, to clearly indicate that the power allocation sets and, thus, the domains of $u_{k}$ are different, we will denote the corresponding utility functions by $v_{k}^{\mathrm{s}}\left(p ; g_{k}\right)$ where $\mathrm{s} \in\{\mathrm{MC}, \mathrm{MT}, \mathrm{SC}\}$ and $\mathrm{MC}, \mathrm{MT}$, and SC respectively stands for multi-channel, multitransmitter, and single-channel scenarios. The single-channel or channel selection scenario corresponds to a network in which every transmitter can use only one channel among $N$ possible ones. Denoting by $P^{\max }$ the maximal transmit power and introducing $p_{k}=\left(p_{k}^{1}, \ldots, p_{k}^{N}\right)$, the sets of power allocation vectors are defined as follows:

- $\mathcal{P}_{k}^{\mathrm{MC}}=\left\{p_{k} \in \mathbb{R}^{N}: p_{k}^{n} \geqslant 0, \sum_{n=1}^{N} p_{k}^{n} \leqslant P^{\max }\right\}$;

- $\mathcal{P}_{k}^{\mathrm{MT}}=\left\{p_{k} \in \mathbb{R}^{N}: p_{k}^{n} \geqslant 0, p_{k}^{n} \leqslant P^{\max }\right\}$;

- $\mathcal{P}_{k}^{\mathrm{SC}}=\left\{P^{\max } e_{1}, \ldots, P^{\max } e_{N}\right\}$ where $\left(e_{1}, \ldots, e_{N}\right)$ represents the canonical basis of $\mathbb{R}^{N}$ (i.e., $e_{1}=(1,0, \ldots, 0), e_{2}=$ $(0,1,0, \ldots, 0)$, etc). An important comment is now in order. The MC and SC scenarios assume a total budget $P^{\max }$ while the MT scenario assumes an $N$ times higher total power budged. In spite of this fact, the latter scenario is of practical importance as well. In the WiFi example mentioned in Section I, channel bundling can be implemented by using available USB ports on the access point and user terminals. In such a case, there can be one radio frequency transmitter per channel and there is no additional power constraint on the sum.

Now, let us define an NE for the games under consideration. With a slight abuse of notation, the power vector $p$ is denoted by $p=\left(p_{1}, \ldots, p_{K}\right)=\left(p_{k}, p_{-k}\right)$.

Definition 1 (Pure Nash Equilibrium). Let $\mathrm{s} \in$ $\{\mathrm{MC}, \mathrm{MT}, \mathrm{SC}\}$. The power vector $p=\left(p_{k}, p_{-k}\right)$ is an NE

\footnotetext{
${ }^{2}$ Convergence is guaranteed under some sufficient conditions.
} 
of the strategic form game $\left(\mathcal{K},\left(\mathcal{P}_{k}^{\mathrm{s}}\right)_{k \in \mathcal{K}},\left(v_{k}^{\mathrm{s}}\right)_{k \in \mathcal{K}}\right)$, if for all $k \in \mathcal{K}$ and for all $p_{k}^{\prime} \in \mathcal{P}_{k}^{\mathrm{s}}$

$$
v_{k}^{\mathrm{s}}\left(p_{k}, p_{-k} ; g_{k}\right) \geqslant v_{k}^{\mathrm{s}}\left(p_{k}^{\prime}, p_{-k} ; g_{k}\right) .
$$

At this point, we can define the different quantities proposed to compare the global performance of an interference network in which transmitters can use several bands simultaneously (i.e., either all transmitters use $\mathcal{P}_{k}^{\mathrm{MC}}$ or they all use $\mathcal{P}_{k}^{\mathrm{MT}}$ ) and another one where using only one band is allowed (i.e., all the transmitters use $\left.\mathcal{P}_{k}^{\mathrm{SC}}\right)$. We will denote by $w^{\mathrm{s}}(p ; g)$ the sum-utility function for scenario $\mathrm{s} \in\{\mathrm{MC}, \mathrm{MT}, \mathrm{SC}\}$.

Definition 2 (Global performance comparison measures). Let $\mathrm{SNR} \triangleq \frac{P^{\max }}{\sigma^{2}}$. The four measures under consideration are as follows:

$$
\begin{aligned}
& \text { - } \operatorname{Pr}_{g}\left[\widetilde{\mathrm{R}}_{\mathrm{S}}(\mathrm{SNR} ; K ; N ; g)<1\right] \text { with } \\
& \qquad \widetilde{\mathrm{R}}_{\mathrm{S}}(\mathrm{SNR} ; K ; N ; g)=\frac{w^{\mathrm{s}}\left(\widehat{p}^{\mathrm{S}}(g) ; g\right)}{w^{\mathrm{SC}}\left(\breve{p}^{\mathrm{SC}}(g) ; g\right)}
\end{aligned}
$$

where $\breve{p}^{\mathrm{SC}}(g)$ is the worst NE in terms of sum-utility of the SC game and $\widehat{p}^{\mathrm{s}}(g)$ is the best $N E$ of the power allocation game $s \in\{\mathrm{MC}, \mathrm{MT}\}$;

- $\operatorname{Pr}_{g}\left[\overline{\mathrm{R}}_{\mathrm{S}}(\mathrm{SNR} ; K ; N ; g)<1\right]$ with

$$
\overline{\mathrm{R}}_{\mathrm{S}}(\mathrm{SNR} ; K ; N ; g)=\frac{w^{\mathrm{s}}\left(p^{\mathrm{UPA}: \mathrm{s}} ; g\right)}{w^{\mathrm{SC}}\left(p^{\mathrm{CS}}(g) ; g\right)}
$$

where $p^{\mathrm{CS}}$ corresponds to the power vector obtained when each transmitter selects its best channel regardless ${ }^{3}$ of the other transmitters' decisions and $p^{\mathrm{UPA}: \mathrm{s}}$ is the power vector obtained when each transmitter allocates the available power uniformly in scenario $s \in\{\mathrm{MC}, \mathrm{MT}\}$. Note that the corresponding power vectors do not always correspond to equilibria;

$$
\widetilde{\Gamma}_{\mathrm{S}}(\mathrm{SNR} ; K ; N)=\frac{\mathbb{E}_{g}\left[w^{\mathrm{s}}\left(\widehat{p}^{\mathrm{s}}(g) ; g\right)\right]}{\mathbb{E}_{g}\left[w^{\mathrm{SC}}\left(\bar{p}^{\mathrm{SC}}(g) ; g\right)\right]}
$$

with the same notations as above;

$$
\bar{\Gamma}_{\mathrm{S}}(\mathrm{SNR} ; K ; N)=\frac{\mathbb{E}_{g}\left[w^{\mathrm{S}}\left(p^{\mathrm{UPA}: \mathrm{s}} ; g\right)\right]}{\mathbb{E}_{g}\left[w^{\mathrm{SC}}\left(p^{\mathrm{CS}}(g) ; g\right)\right]}
$$

with the same notations as above.

The above quantities rely on the equilibrium analysis of the power allocation games under consideration. The corresponding analyses have been conducted in [11][6][9] and will not be detailed here. The main points to be mentioned are as follows. The power allocation game for the scenario MC possesses in general several equilibria [6]. The same observation holds for the channel selection game for scenario SC [9]. This is the reason why the best and worst equilibria are considered. In fact, the metric $\widetilde{R}_{\mathrm{S}}$ is deliberately in favor of the power allocation game MC. If the best equilibrium of the game in scenario SC is considered, the global performance degradation phenomenon under consideration occurs more frequently;

\footnotetext{
${ }^{3}$ More details about this policy are provided in Section III.
}

some numerical results will be provided to illustrate this point. Now, in the power allocation game of scenario MT, there exists a unique Nash equilibrium which corresponds to using full power on each band. Therefore, the equilibrium power allocation policies can be seen as uniform power allocation policies with a power budget of $N P^{\max }$.

The main purpose of the next two sections is to exploit the above measures to prove the existence of scenarios where allowing every transmitter to use several bands instead of one leads to a global performance degradation. These scenarios correspond to a large extent to asymptotic regimes. It turns out that some of those regimes can be observed in practice; operating SNRs are (resp. will be) typically high in WiFi networks (resp. SCNs).

\section{ANALYTICAL PERFORMANCE COMPARISON}

In Section II, we have implicitly assumed (without loss of generality) that the noise level is the same at all the receivers. The common SNR parameter SNR $=\frac{P^{\max }}{\sigma^{2}}$ plays a major role in determining whether using multiple bands instead of one is beneficial in terms of global performance. When the SNR is small, equilibrium power allocation policies in scenarios SC and $\mathrm{MC}$ can be proved to coincide.

Proposition 1 (Low SNR regime, $K, N$ arbitrary). For all $K \geqslant 2, N \geqslant 2$, and $\mathrm{s} \in\{\mathrm{MC}, \mathrm{MT}\}$ we have that

$$
\lim _{\mathrm{SNR} \rightarrow 0} \operatorname{Pr}_{g}\left[\widetilde{\mathrm{R}}_{\mathrm{S}}(\mathrm{SNR} ; K ; N ; g)<1\right]=0 .
$$

The proof is omitted because of space limitations. Proposition 1 translates that the interference is negligible compared to the noise when $\sigma^{2} \rightarrow \infty$. The transmitters' decisions are no longer interdependent and the power allocation games MC, MT, and SC become classical optimization problems: only the direct channel gains $g_{k k}^{n}$ matter to transmitter $k$. Since total transmit power is limited to $P^{\max }$ for $\mathrm{MC}$, the rate is maximized by selecting the best band. The SC game also becomes a mere channel selection problem, which means that the distributed power allocation policies of MC and SC coincide at low SNR. On the other hand, for the game MT, the point $p=\left(P^{\max }, \ldots, P^{\max }\right) \in \mathbb{R}^{K N}$ remains the unique Nash equilibrium and its structure does not change with the operating SNR. This equilibrium can be checked to be more sum-rate-efficient than the equilibrium of the SC game, explaining that the above probability vanishes with the SNR.

A natural question is to know what happens when the SNR is high. Do we reach the same conclusion as in the low SNR regime? Providing the general answer for an arbitrary pair $(K, N)$ seems to be a non-trivial problem, which is left as an extension of this paper. Rather, we treat here some special cases of interest $N \geqslant K$ and $K=N=2$ that provide some useful insight into the general case.

Proposition 2 (High SNR regime, $K \leqslant N$ ). Consider a symmetric interference channel where $\forall n, \gamma_{11}^{n}=\gamma_{22}^{n}=\lambda$, 
$\gamma_{12}^{n}=\gamma_{21}^{n}=\mu$, and $\frac{\lambda}{\mu}<\infty$. Assuming $K \leqslant N$, we have that

$$
\lim _{\mathrm{SNR} \rightarrow \infty} \operatorname{Pr}_{g}\left[\widetilde{\mathrm{R}}_{\mathrm{MC}}(\mathrm{SNR} ; 2 ; 2 ; g)<1\right]=\omega\left(\frac{\lambda}{\mu}\right),
$$

with $\omega(x) \triangleq\left(1-\frac{1}{(1+x)^{2}}\right)^{2}$;

$$
\lim _{\mathrm{SNR} \rightarrow \infty} \operatorname{Pr}_{g}\left[\widetilde{\mathrm{R}}_{\mathrm{MT}}(\mathrm{SNR} ; K ; N ; g)<1\right]=1 .
$$

For the scenario MC, it is seen that (in the high SNR regime) allowing the transmitters to water-fill over the $N$ available bands instead of restricting the spectrum use to a single band leads to a global performance degradation with a probability which is not vanishing. The probability of having a performance degradation is seen to be an increasing function of $\frac{\lambda}{\mu}$. It equals to $\frac{9}{16}=56.25 \%$ when the channels gains are i.i.d. (namely, $\lambda=\mu$ ) and equals about $98 \%$ when $(\lambda, \mu)=(1,0.1)$; note that this result holds for high SNR. Simulations will show that, for medium SNRs, the considered probability is in fact decreasing from a certain SNR value, confirming the intuition that under low interference levels water-filling is optimal. The result concerning the potential performance degradation questions the use of algorithms such as the IWFA; rather, an iterative algorithm which operates with discrete sets may lead to a better global performance. At high SNR, the scenario MT, in which equilibrium consists in using all bands at full power, always performs less than the worst equilibrium of the scenario SC. This is due to the fact the global performance is interference limited in this regime and the MT equilibrium creates more interference than the MC equilibrium, which is already less efficient than the SC equilibrium with a typically high probability. Of course, the fact that the degradation event is likely in scenario MC does not necessarily mean that the average rate is severely degraded, which explains why the expected rate is also considered. This is the purpose of the next proposition.

Proposition 3 (Extreme SNR regimes, expected rates). Assume that $\forall n, \gamma_{11}^{n}=\gamma_{22}^{n}=\lambda, \gamma_{12}^{n}=\gamma_{21}^{n}=\mu$, and $\frac{\lambda}{\mu}<\infty$. We have that

$$
\begin{aligned}
& \lim _{\mathrm{SNR} \rightarrow 0} \widetilde{\Gamma}_{\mathrm{MC}}(\mathrm{SNR} ; K ; N)=1, \text { for arbitrary Kand } N ; \text { (6) } \\
& \lim _{\mathrm{SNR} \rightarrow 0} \widetilde{\Gamma}_{\mathrm{MT}}(\mathrm{SNR} ; K ; N)=\frac{N}{\sum_{n=1}^{N} \frac{1}{n}} \text {, for arbitrary Kand } N ; \\
& 1-\omega\left(\frac{\lambda}{\mu}\right)<\lim _{\mathrm{SNR} \rightarrow \infty} \widetilde{\Gamma}_{\mathrm{MC}}(\mathrm{SNR} ; 2 ; 2)<1-\frac{1}{2} \omega\left(\frac{\lambda}{\mu}\right)
\end{aligned}
$$

where $\omega(x)$ is defined in Proposition 2;

$$
\lim _{\mathrm{SNR} \rightarrow \infty} \widetilde{\Gamma}_{\mathrm{MT}}(\mathrm{SNR} ; K ; N)=0, \text { for any } K \leqslant N .
$$

The first result translates that the equilibrium power allocation policies of the MC and SC scenario coincide in terms of expected rate at low SNR. The second result allows one to quantify to what extent MT equilibrium policies performs better than SC ones. This gain is seen to be independent of $\lambda$ and $\mu$, which is due to the fact that noise dominates the interference and useful or direct channels are identically distributed. The third result shows that equilibrium MC policies necessarily induce a performance degradation and the corresponding loss can be bounded. In a typical case in which the interference power is smaller than the useful signal by $10 \mathrm{~dB}$ (e.g., when $(\lambda, \mu)=(1,0.1)$ ), we have that $0.02<\widetilde{\Gamma}_{\mathrm{S}}(\mathrm{SNR} ; 2 ; 2)<0.51$. This means that using a multichannel power allocation scheme instead of a single-channel one leads to dividing the total sum-rate by at least $\frac{1}{0.51} \simeq 2$. The fourth result readily follows from the result of Proposition 2 concerning the MT scenario.

So far, almost all the provided results concern finite interference networks regarding the number of transmitter-receiver pairs and the number of bands. An important question is whether the exhibited performance degradation phenomenon can be observed in larger networks. Finding the worst and best equilibria in games with large number of players does not seem to be trivial and is left as a significant extension of the present work. Instead, we compare interesting power allocation policies which do not correspond to equilibria in general:

- For the MC scenario: $\forall k \in \mathcal{K}, p_{k}=\left(\frac{P^{\max }}{N}, \ldots, \frac{P^{\max }}{N}\right)$.

- For the SC scenario: $\forall k \in \mathcal{K}, p_{k}=P^{\max } e_{n_{k}^{\star}}$ where $n_{k}^{\star}=\arg \max _{n}^{n}$.

- For the MT scenario: $\forall k \in \mathcal{K}, p_{k}=\left(P^{\max }, \ldots, P^{\max }\right)$. The uniform power allocation policy happens to be the unique equilibrium of the game MT as well.

Assuming these policies and a large interference network, the following result can be proved.

Proposition 4 ( $K$ large, $N$ finite). Assume $K \rightarrow \infty, N<\infty$ with i.i.d. information channel gains $g_{k k}^{n}$ and i.i.d interference channel gains $g_{k \ell}^{n}(k \neq \ell)$. Then for all $\mathrm{s} \in\{\mathrm{MC}, \mathrm{MT}\}$ we have that

$$
\lim _{\mathrm{K} \rightarrow \infty} \overline{\mathrm{R}}_{\mathrm{S}}(\mathrm{SNR} ; K ; N ; g)=\ell_{N}=\frac{1}{\sum_{n=1}^{N} \frac{1}{n}}
$$

If $K \rightarrow \infty, N \rightarrow \infty$ and $\frac{N}{K} \rightarrow 0$, we have that $\ell_{N} \sim \frac{1}{\log N}$.

This result allows one to compare the scaling laws of two interference networks which allow only uniform power allocation and channel selection respectively. For $N=10$, the corresponding ratio equals about 3 , showing a significant degradation involved by allowing the transmitter to exploit all the available bands. Of course, this result is pessimistic in the sense that interference channels are assumed to have the same average gain as the direct channels. Otherwise, the interference scenario will be less severe. The generalization of Proposition 4 to the non i.i.d. channel gains may be obtained by using tools from large random matrix theory [12] [13]. It might be argued that the number of transmitter-receiver pairs is taken to be large while not expanding the available spectrum resources. To address this issue another type of asymptotic regimes has 
to be considered that will not be be tackled here.

\section{NumERiCAL RESUlTS}

Finite systems: Multi-Channel scenario equilibria vs. Single-Channel scenario equilibria. We consider here that $(K, N)=(2,2)$ and $(K, N)=(4,4)$. The direct channels are assumed stronger than the interfering ones: $(\lambda, \mu)=$ $(1,0.1)$. Our objective is to evaluate the sum-rate performance gap between the $\mathrm{MC}$ and $\mathrm{SC}$ at equilibrium. Fig. 1 and 2 respectively represent $\operatorname{Pr}_{g}\left[\widetilde{\mathrm{R}}_{\mathrm{MC}}(\mathrm{SNR} ; K ; N ; g)<1\right]$ and $\widetilde{\Gamma}_{\mathrm{MC}}(\mathrm{SNR} ; K ; N)$ as functions of $\mathrm{SNR}(\mathrm{dB})=10 \log _{10} \mathrm{SNR}$. As our analytical results forecast, the probability that the $\mathrm{MC}$ equilibrium power allocation policies perform less than the SC ones tends to zero at low SNR and tends to one at high SNR. Fig. 1 also shows that this probability increases with SNR. These results are obtained using the IWFA algorithm under the equilibrium uniqueness condition of [11]. Interestingly, the figure allows one to delineate the two regimes in which the multi-channel solution performs better or worse than the SC one: the SNR threshold is about $25 \mathrm{~dB}$. Above this threshold, restricting the choices of the transmitters in terms of using the spectrum is beneficial. For example, as shown in Fig. 2, when $\mathrm{SNR}(\mathrm{dB})=45 \mathrm{~dB}$ (which is a typical value in WiFi systems and will also be quite common in SCNs) the equilibrium MC sum-rate is only $60 \%$ of the sum-rate achieved by allowing the transmitters to use a single band only. Since these observations hold for a typical but specific choice $(\lambda, \mu)=(1,0.1)$, we also study the influence of the ratio $\frac{\lambda}{\mu}$ which represents the relative strength of the useful link compared to interference links. For $\mathrm{SNR}(\mathrm{dB})=70 \mathrm{~dB}$ and $\lambda=1$, Fig. 3 precisely represents $\operatorname{Pr}_{g}\left[\widetilde{\mathrm{R}}_{\mathrm{MC}}(\mathrm{SNR} ; K ; N ; g)<1\right]$ as function of $\frac{\lambda}{\mu}$ in $\mathrm{dB}$, for $(K, N)=(2,2)$ and $(K, N)=(4,4)$. As proved in (4) for $(K, N)=(2,2)$, it can be seen that the probability that MC induces global performance degradation increases with the latter ratio. In other words, if the interference is relatively weak, the probability of performance degradation will be high. This is also confirmed by simulations for $(K, N)=(4,4)$. But this holds for very high SNRs. By considering $\mathrm{SNR}(\mathrm{dB})=50$ dB, Fig. 4 shows that the performance degradation vanishes as the interference level decreases. The intuition is that, when the interference is low, the network behaves like a set of independent single-user communications i.e., water-filling over all the available bands is optimal. However, this is not observed for $\mathrm{SNR}(\mathrm{dB})=70 \mathrm{~dB}$ and higher values for SNR: when $\sigma^{2} \rightarrow 0$, the interference is not negligible compared to the noise. Other simulations, which are not reported here for obvious space limitations confirm the general tendencies the three commented figures indicate for different $(K, N)$.

Finite systems: Multi-Transmitter scenario equilibria vs. Single-Channel scenario equilibria. Here, exactly the same approach as the preceding subsection is conducted by considering the MT scenario instead of MC. The observations concerning $\operatorname{Pr}_{g}\left[\widetilde{\mathrm{R}}_{\mathrm{MT}}(\mathrm{SNR} ; K ; N ; g)<1\right]$ are similar to those made for $\operatorname{Pr}_{g}\left[\widetilde{\mathrm{R}}_{\mathrm{MC}}(\mathrm{SNR} ; K ; N ; g)<1\right]$ and will therefore

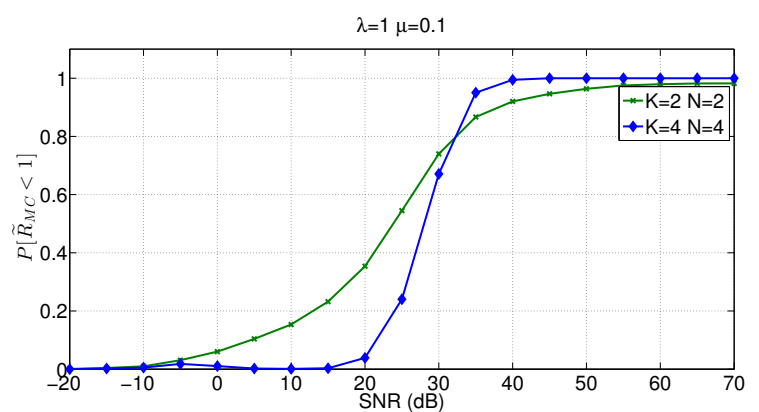

Fig. 1: Probability that using multiple channels (MC) instead of one (SC) induces a global performance degradation at equilibrium vs. SNR.

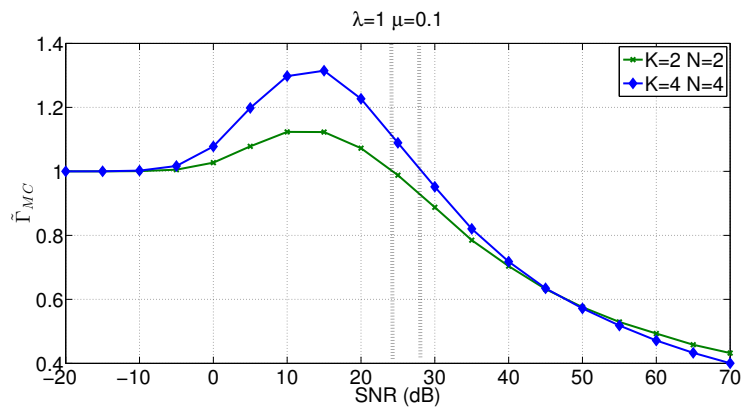

Fig. 2: Ratio between the expected equilibrium sum-rates in $\mathrm{MC}$ and $\mathrm{SC}$ scenarios.

not be reported here. For $\widetilde{\Gamma}_{\mathrm{MT}}(\mathrm{SNR} ; K ; N)$ the behavior is also quite similar to $\widetilde{\Gamma}_{\mathrm{MC}}(\mathrm{SNR} K K N)$, as advocated by Fig. 5. For $(K, N)=(4,4)$, it can be seen that: at low SNR, using the multi-transmitter solution allows the sum-rate to be multiplied by about 2 w.r.t. the single-channel solution; at high SNR, the MT solution sum-rate is divided by about 2; for intermediate SNR, the sum-rate performance of the MT and SC are close. At low SNR, the values forecasted by (7) are validated. Similarly, when the SNR goes beyond $70 \mathrm{~dB}$, predictions from (9) are also observed.

Large systems: MC/MT Uniform Power Allocation policy vs. Best Channel Selection policy. We focus here on the expected sum-rate comparison between the UPA (MC setting), the full power $P^{\max }$ in every channel policy (MT setting) and the best channel selection policy (CS setting). Fig. 6 represents the expected sum-rates against the number of transmitterreceiver pairs $K$, for the scenario: $N=10, \mathrm{SNR}=10$ $\mathrm{dB},(\lambda, \mu)=(1,0.1)$. This figure allows one to have an idea about the network scaling laws under different power allocation policies. Roughly, when the system is not loaded, say for a load which is less than $\frac{K}{N}=\frac{30}{10}$ for the considered simulation setting, using several bands is beneficial for the network sum-rate. Above this threshold, using a single band allows to achieve a significantly better sum-rate performance.

\section{CONCLUSION}

In distributed interference networks, allowing individual rate-maximizing transmitters to spread their power over the 


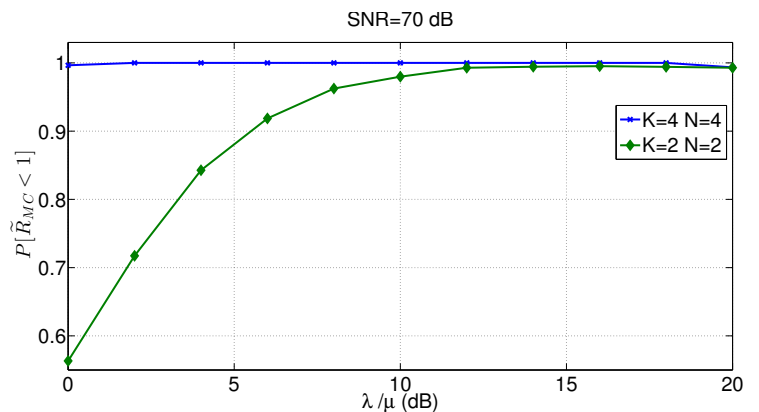

Fig. 3: Probability that using multiple channels (MC) instead of one (SC) induces a global performance degradation at equilibrium vs. $\frac{\lambda}{\mu}$.

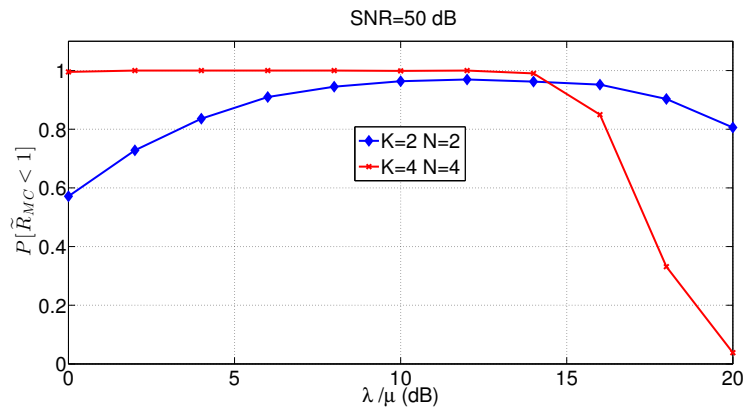

Fig. 4: Probability that using multiple channels (MC) instead of one (SC) induces a global performance degradation at equilibrium vs. $\frac{\lambda}{\mu}$.

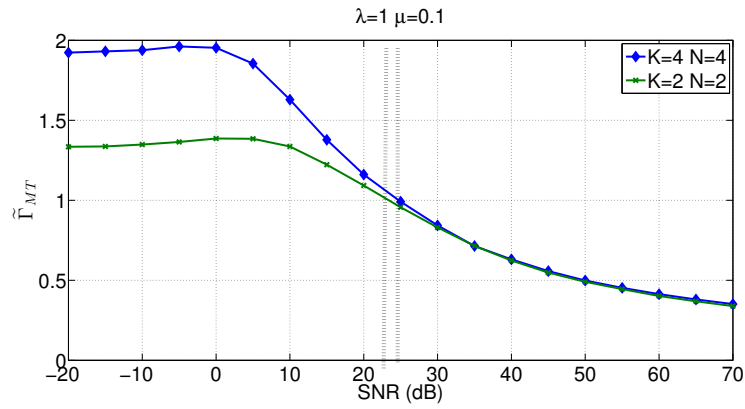

Fig. 5: Ratio between the expected equilibrium sum-rates in MT and SC scenarios.

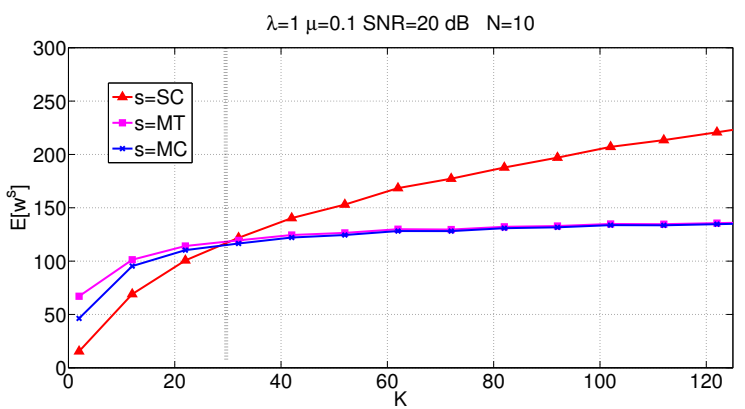

Fig. 6: Expected sum-rate against the number of users $K$. entire spectrum, as opposed to using a single band, may result in sum-rate performance losses. In this work, three spectrum allocation scenarios are compared in terms of equilibrium sumrates: multi-channel (the users can spread their powers over several channels under an overall power constraint), multitransmitter (the users can spread their power under a different power constraint per channel), and single-channel scenarios (the users can only choose one channel). These scenarios differ only in the available transmitters' action choices. At low SNR, when noise dominates interference the multi-channel and single-channel are shown to perform similarly while the multi-transmitter dominates both of them thanks to the fact more available total power is available $\left(N P^{\max }\right.$ instead of $\left.P^{\max }\right)$. At high SNR, the single-channel solution is shown to outperform both the multi-channel and multi-transmitter solutions. The same observation is made when increasing the number of users for a fixed SNR; using a single channel instead of multiple channels is very beneficial in terms of sumrate. Simulation results validate the proved results and allow one to quantify the thresholds in terms of SNR and number of users which delineate the regimes in which the singlechannel dominates (or is dominated by) the multi-channel and multi-transmitter solutions. Therefore, our results question the use of distributed power allocation algorithms such as the IWFA. Possible extensions include: interference channels with more diverse channel gains statistics and correlation profiles, multiple input and multiple output systems.

\section{REFERENCES}

[1] http://www.codeontechnologies.com/technology/demos-andsimulator/multipath-demo/

[2] J. Hoydis, M. Kobayashi, and M. Debbah, "Green Small-Cell Networks," IEEE Vehicular Technology Magazine, vol. 6, no. 1, pp. 37-43, Mar 2011.

[3] A. J. Goldsmith, and S. B. Wicker, "Design challenges for energyconstrained ad hoc wireless networks," IEEE Trans. Wireless Commun., vol. 9, no. 4, pp. 8-27, Aug. 2002.

[4] S. Haykin, "Cognitive radio: brain-empowered wireless communications," IEEE J. Sel. Areas Commun., vol. 23, no.2, pp. 201-220, Feb. 2005

[5] S. Lasaulce, and H. Tembine, "Game theory and learning for wireless networks," Elsevier Academic Press, Aug. 2011.

[6] L. Rose, S. M. Perlaza, and M. Debbah, "On the Nash equilibria in decentralized parallel interference channels," IEEE Intl. Conf. on Communications (ICC), pp. 1-6, Kyoto, Japan, Jun. 2011.

[7] E. Telatar, "Capacity of multi-antenna Gaussian channels," European transactions on telecommunications, vol. 10, no. 6, pp. 585-595, 1999.

[8] T. M. Cover, and J. A. Thomas, "Elements of Information Theory," New York: Wiley, 2006.

[9] S. M. Perlaza, S. Lasaulce, and M. Debbah,"Equilibria of channel selection games in parallel multiple access channels," EURASIP J. on Wireless Commun. and Networking, vol. 2013, no. 15, pp. 1-23, Jan. 2013.

[10] G. Scutari, D. P. Palomar, and S. Barbarossa, "Competitive design of multiuser MIMO systems based on game theory: A unified view," IEEE J. Sel. Areas Commun., vol. 26, no. 7, pp. 1089-1103, Sep. 2008.

[11] W. Yu, G. Ginnis and J. Cioffi, "Distributed multiuser power control for digital subscriber lines," IEEE J. Sel. Areas Commun., vol. 20, no. 5, pp. 1105-1115, Jun. 2002.

[12] R. Couillet, and M. Debbah, "Random matrix methods for wireless communications," Cambridge University Press, Sep. 2011.

[13] J. Dumont, W. Hachem, S. Lasaulce, P. Loubaton and J. Najim, "On the capacity achieving covariance matrix for Rician MIMO channels: an asymptotic approach," IEEE Trans. Inf. Theory., vol. 56, no. 3, pp. 10481069, Mar. 2010 